\title{
Reamed interlocking intramedullary nailing for the treatment of tibial diaphyseal fractures and aseptic nonunions. Can we expect an optimum result?
}

\author{
Byron E. Chalidis · George E. Petsatodis • \\ Nick C. Sachinis · Christos G. Dimitriou • \\ Anastasios G. Christodoulou
}

Received: 7 February 2009/Accepted: 4 August 2009/Published online: 25 August 2009

(C) Springer-Verlag 2009

\begin{abstract}
The need for reaming and the number of locking screws to be used in intramedullary (IM) tibial nailing of acute fractures as well as routine bone grafting of tibial aseptic nonunions have not been clearly defined. We describe the results of reamed interlocked IM nails in 233 patients with 247 tibial fractures (190 closed, 27 open and 30 nonunions). Ninety-six percent of the fractures were united at review after an average of 4.9 years. No correlation was found between union and nail diameter $(P=0.501)$ or the number of locking screws used $(P=0.287)$. Nail dynamization was effective in $82 \%$ of fractures. Locking screw(s) breakage was associated with nonunion in $25 \%$ of cases. Bone grafting during IM nailing was found not to increase the healing rate in tibial nonunions $(P=0.623)$. None of the IM nails were removed or revised due to infection. A dropped hallux and postoperative compartment syndrome were found in 0.8 and $1.6 \%$ of cases, respectively. Anterior knee pain was reported in $42 \%$ of patients but nail removal did not alleviate the symptoms in almost half. This series confirms the place of reamed intramedullary nailing for the vast majority of tibial diaphyseal fractures. It provides an optimum outcome and minimizes the need for supplementary bone grafting in aseptic nonunions.
\end{abstract}

\footnotetext{
B. E. Chalidis - G. E. Petsatodis · N. C. Sachinis ·

C. G. Dimitriou - A. G. Christodoulou

1st Orthopaedic Department of Aristotle,

University of Thessaloniki, Thessaloniki, Greece

B. E. Chalidis $(\square)$

31 Filippou St, 54631 Thessaloniki, Greece

e-mail: byronchalidis@gmail.com
}

Keywords Tibia Fracture Open fracture . Intramedullary nail $\cdot$ Nonunion $\cdot$ Dynamization . Exchange nailing

\section{Introduction}

Intramedullary (IM) nailing is a popular technique for the fixation of both closed and open tibial fractures [1]. Intramedullary reaming allows the insertion of largerdiameter and tighter-fitting nails which offer better bending and rotational stability [2]. In addition, insertion of locking screws at both ends of the nail increases the biomechanical strength of the construct as it provides higher resistance to axial and torsional forces [3]. However, the exact endpoint of the reaming process, the necessity of widening the intramedullary canal in all fracture types, the efficacy of nail dynamization and the optimal number of locking screws on each side of the nail are factors that have not been clearly established. Furthermore, there is limited data regarding the benefit of supplementary bone grafting or use of fracture healing stimulants (osteoinductive factors) during nailing of aseptic nonunions.

The purpose of this study was to evaluate the efficacy of reamed interlocked IM nailing in acute diaphyseal tibial fractures and nonunions. The role of nail diameter, number of locking screws, nail dynamization and supplementary bone grafting in tibial nonunions were also investigated.

\section{Materials and methods}

Between 1996 and 2005, 246 patients with 261 tibial fractures were treated in our hospital with antegrade interlocked intramedullary nailing. Thirteen patients 
(14 fractures) were lost to follow-up due to death, relocation or other reasons, leaving 233 patients with 247 tibia fractures for investigation.

There were 190 closed fractures, 27 open fractures and 30 nonunions. The fractures were described according to the Muller classification [4] and open fractures were additionally classified by the Gustilo and Anderson system [5]. The average follow-up was 4.9 years (range, 2-9 years).

The fractures were managed with patients in supine position on a fracture table. A thigh bolster was routinely applied and a tourniquet was inflated in 210 out of 247 cases. The knee was flexed but no calcaneal traction was used for fracture reduction. A patellar tendon-splitting (transtendinous) approach was selected in all cases. Guide wire insertion was followed by sequential reaming in 0.5 $\mathrm{mm}$ increments until the canal was reamed up to $1 \mathrm{~mm}$ greater than the selected diameter of the cannulated stainless steel tibial nail (Russel-Taylor, Smith \& Nephew, Memphis, TN). All the nails were introduced with a snug fit to the intramedullary cavity and were statically locked. The nail design allowed the insertion of a maximum of two screws proximally and two screws distally. However, the decision to use one or two screws at each side of the nail was made at the discretion of the attending surgeon.

In open fractures, urgent wound irrigation and thorough debridement preceded nail insertion. Wound closure was performed either primarily within $24 \mathrm{~h}$ or by secondary intention according to soft tissues status. In general, intravenous cefuroxime was used as infection prophylaxis for 1 day in closed fractures whilst, in Gustilo Types I and II fractures, the time period was extended to $48 \mathrm{~h}$. In Gustilo Type IIIA fractures, intravenous gentamicin was added and the total duration of IV antibiotics administration was increased to $72-96 \mathrm{~h}$.

The operation technique for the treatment of tibial nonunions consisted of removal of the old implant and subsequent IM nailing with or without additional bone grafting (iliac crest autograft and/or allograft). The graft was inserted through a small incision at the nonunion site after removal of the fibrous tissue and proper bone decortication. If the case was one of previous IM nailing, the canal was reamed further and the exchange nail used was at least $1 \mathrm{~mm}$ larger in diameter than the nail being removed.

Nail dynamization was carried out at least 10-12 weeks postoperatively when slow or no progression to bone healing was observed. Under local anaesthesia, the farthest screw(s) from the fracture site were removed; the aim was to stimulate the local osteogenic activity through increase in axial motion.

The fracture was considered to be healed when radiographic evidence of bridging callus formation was seen across three cortices in the two X-ray views. The absence of either adequate fracture callus after a minimum period of 9 months or progression towards healing for 3 consecutive months was defined as a nonunion. Delayed union was assumed when no sign of union was seen at 2026 weeks postoperatively.

This study was approved by the Ethics Committee of the hospital.

\section{Statistical analyses}

Statistical evaluation was carried out with use of the SPSS software package (SPSS 12.0, Chicago, Illinois). The effect of nail diameter or the number of locking screws on union rate was examined with the Chi-squared test. The same test was conducted to explore the effect of supplementary bone grafting in aseptic tibial nonunions. $P$ values of less than 0.05 were considered to be statistically significant.

\section{Results}

There were 175 men $(71 \%)$ and 58 women (29\%) with a mean age of 38.9 years (range 17-95 years). The left limb was affected in 134 cases $(54 \%)$ and the right one in 116 cases $(46 \%)$. The fracture showed a higher prevalence among men in all age groups particularly during the second decade of life. A motor-vehicle accident was the main mechanism of injury in almost half of the patients (117 fractures $=48 \%)$. A fall from a height (67 fractures $=27 \%)$, a direct blow $(60$ fractures $=(24 \%)$ and labour accidents ( 3 fractures $=1 \%$ ) were also recorded as mechanisms. The average hospitalization time was 10 days (range 4-27 days).

There were 118 Type A (48\%), 90 Type B (36\%) and 39 Type C (16\%) fractures. One hundred sixteen fractures $(47 \%)$ were located in the middle third of the tibial shaft, 11 fractures (4\%) in proximal third and 120 fractures $(49 \%)$ in distal third. The majority of open fractures were Type I (18 fractures) and a few were Type II (3 fractures) or Type IIIA (6 fractures). None of the Types IIIB or IIIC fractures were treated primarily by the described method.

Among the 30 tibial nonunions, 17 were hypertrophic (57\%) and $13(47 \%)$ atrophic. There were no significant areas of bone loss or segmental defects of more than $1 \mathrm{~cm}$ present. These fractures were initially treated with external fixation (14 cases), IM nailing (9 cases), plates and screws ( 4 cases) or casts ( 3 cases) (Fig. 1). The average time from the primary intervention to the index procedure was 15 months (range 5-54 months). In 12 out of 30 cases, additional bone grafting (iliac crest autograft and/or allograft) was used. 
Fig. 1 a External fixation of a Type IIIA open tibial fracture in a 36-year-old man. b 15 months after the index procedure the fracture has not united. c Interlocked IM nailing without bone grafting was performed at that time. d At 4 months postoperatively the fracture was healed
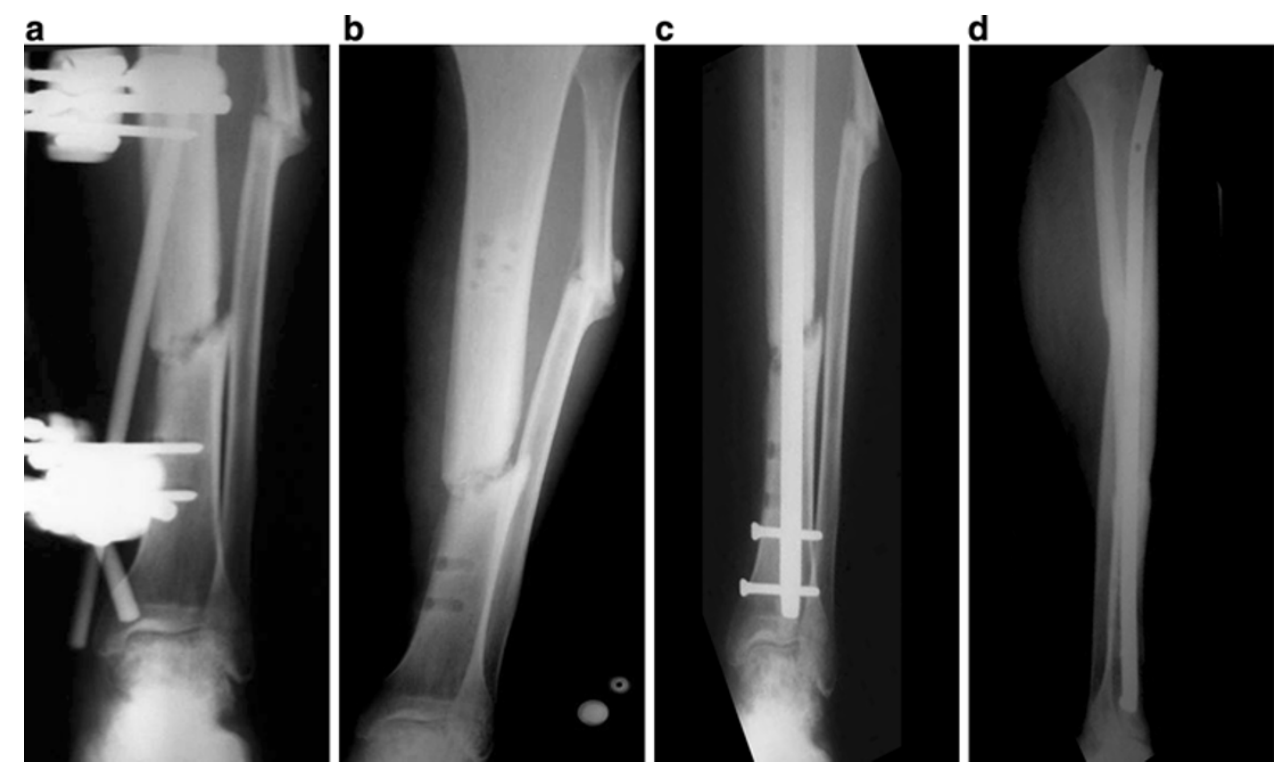

The average nail width was $11 \mathrm{~mm}$ (range 8-13). Fortyone nails were locked with two screws, 97 with three screws and 109 with four screws.

\section{Union}

There were 225 fractures (91\%) that united, 12 fractures $(5 \%)$ showed delayed union and 10 nonunions $(4 \%)$. The last category included one open fracture (Gustilo Type II), six closed fractures (4 Type C and 2 Type B) and three prior non-unions (Fig. 2). The mean time to union was 3.2 months (range 1-8 months). No relationship was found between union and nail width $(P=0.501)$ or the number of locking screws $(P=0.287)$ used. Supplementary grafting in tibial nonunions did not influence the incidence of facture healing $(P=0.623)$ in this series.

The tibial nail was dynamized in 22 cases (9\%). In 18 of these 22 cases $(82 \%)$, the method was associated with fracture union (Fig. 3).

\section{Complications}

Compartment syndrome requiring fasciotomy developed in one patient preoperatively and in four patients $(1.6 \%)$ postoperatively. In two of the above cases, a fasciocutaneous flap was deemed necessary for secondary wound closure.
Fig. 2 a Gustilo Type II open tibial fracture in a 49-year-old man. b 6 months after the insertion of the interlocked IM nailing no fracture union was observed. c The same result was evident at 14 months postoperatively a

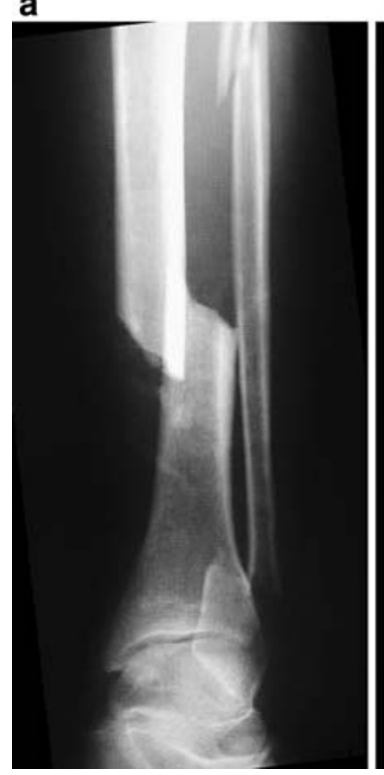

b

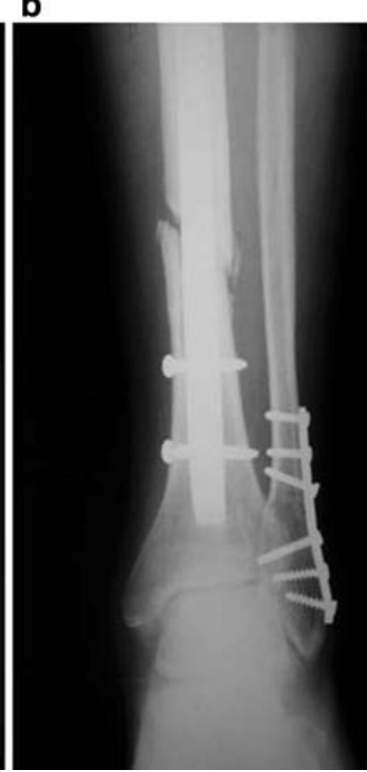

C

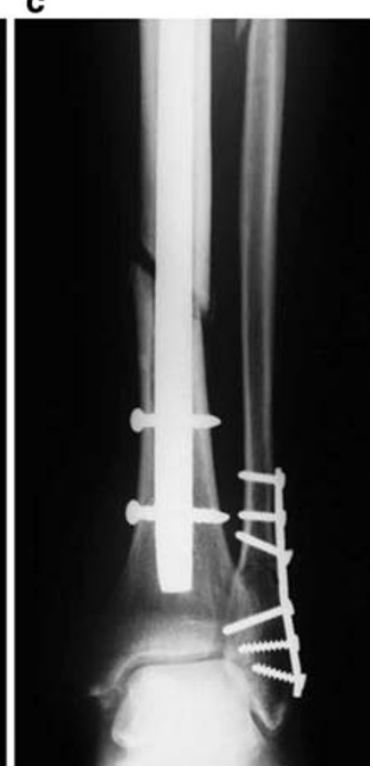



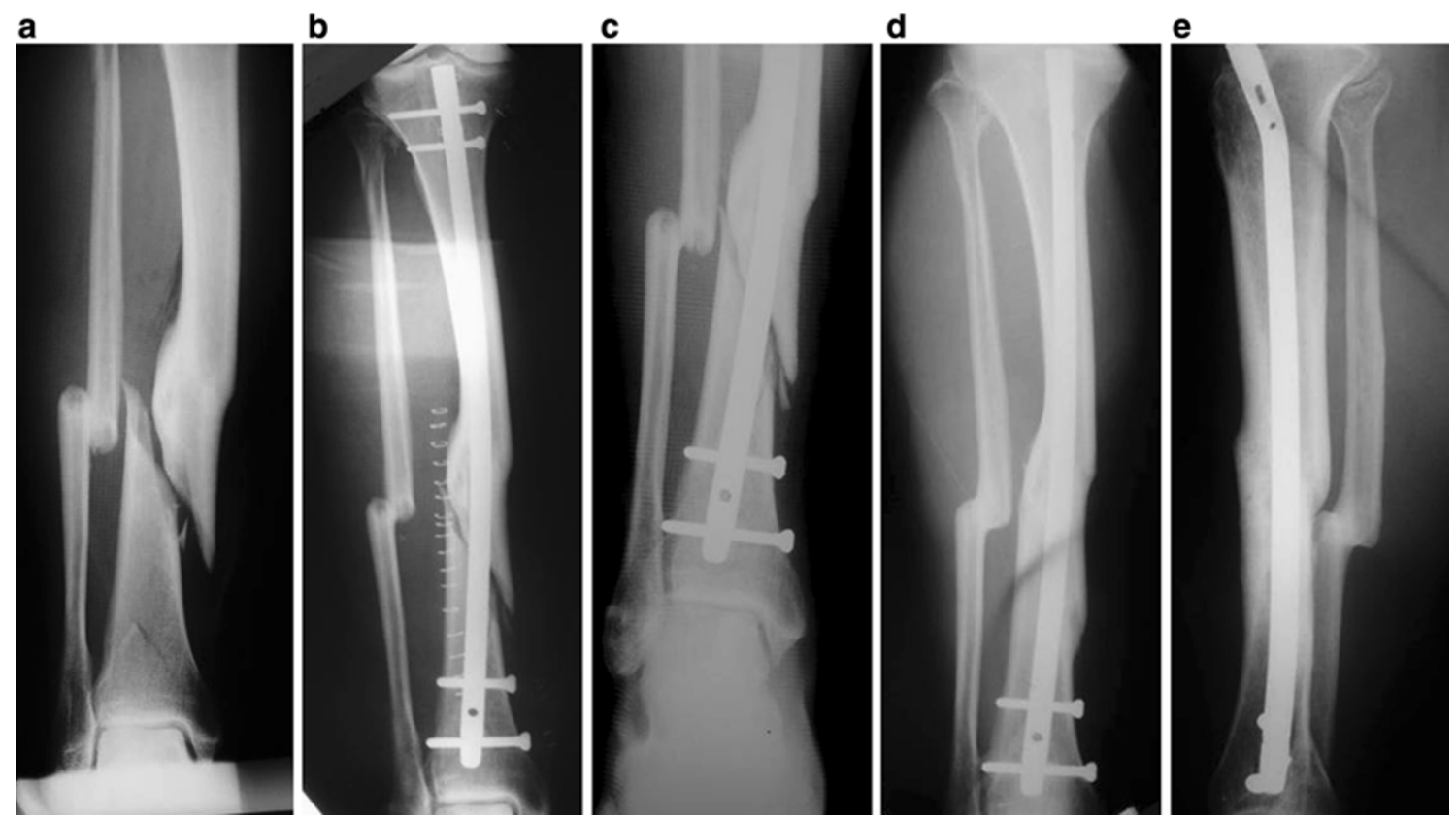

Fig. 3 a Closed tibial fracture at the site of a previous malunion in a 50 -year-old man. The first fracture was treated conservatively 25 years ago. b Slight bending of the IM nail during its insertion was noted intraoperatively. c After 4 months, dynamization of the

A dropped hallux without peroneal nerve dysfunction was noted in another two patients $(0.8 \%)$. As no marked improvement occurred postoperatively, an exploration of the flexor hallucis longus musculotendinous area was performed after 9 and 12 months, respectively. Scar tissue formation along the muscle belly was the main finding and lengthening of the tendon was subsequently performed. In both cases the fractures were closed and a tourniquet was used.

Superficial infection was diagnosed in five cases $(2 \%)$ and IV antibiotics were used. No wound debridement or implant removal was required.

Screw breakage was observed in 15 cases $(6.2 \%)$ but only in 4 cases did this lead to failure of union. In another 27 patients (11\%), isolated screw(s) removal was performed due to painful prominence of screw head.

Mild or moderate knee pain, exacerbated by knee flexion and kneeling, was reported by 103 (42\%) patients. At the latest follow-up visit, 73 nails (30\%) were removed either electively (29 cases) or for knee pain (44 cases). However, 19 patients in the last group (43\% of the removals) were still complaining of residual pain despite the improvement of knee function.

\section{Discussion}

Intramedullary nailing is a well established surgical technique for the treatment of closed and the majority of open nail by removal of proximal screws was performed due to inadequate callus formation. d, e At 8 months postoperatively the fracture was united

tibial fractures. It is associated with union rates of up to 96-100\% [1, 6, 7]. There are studies with level 1 evidence (well designed prospective randomized controlled trials) demonstrating a positive effect of tibial reaming to the union rate and time to union. Blachut et al. [1] described 96 and $89 \%$ union rates among reamed and unreamed groups accordingly. Finkemeier et al. [2] found a statistically significant difference in union time in favour of reamed nails during the first 4 postoperative months. Similarly, Larsen et al. [7] described no nonunions in the reamed group (22 patients) while three fractures in the unreamed group (23 patients) failed to be healed. The mean time to union was 16.7 weeks for reamed nails and 25.7 weeks for unreamed nails $(P=0.004)$.

The potential impairment of bone vascularity during tibial reaming has been a topic of research during the last two decades. Klein et al. [8] in a canine study demonstrated that the reaming process may destroy almost $70 \%$ of the endosteal blood flow. Schemitsch et al. [9] noted in a sheep model that stabilization of a spiral tibial fracture with an unreamed nail led to revascularization of the tibial cortex in 6 weeks. When the bone canal was reamed the time increased to 12 weeks. However, in their later experimental study, using the same animal model, they found no difference in new bone formation between reamed and unreamed nails. This finding might be attributed to increased periosteal circulation after reaming which compensates for any reduction in endosteal blood flow, and the 
osteoblast proliferation as a result of local limb ischaemia [10]. Moreover, the products of reaming contain osteoblasts [11] and multipotent stem cells [12] which can act as an effective bone graft of high osteogenic potential [13]. According to published clinical studies, widening of tibial canal does not seem to have any detrimental effect on fracture union and may be advantageous in promoting fracture healing [13].

The role of reaming in open tibial fractures is still controversial due to the risk of infection which may increased to $50 \%$ in open IIIb tibial fractures [6]. Keating et al. [14] and Finkemeier et al. [2] found no difference in union rate, time to healing and incidence of infection between reamed and unreamed nails in open tibial fractures. Bhandari et al. [15], taking into account the data of from both studies, noticed only a weighted absolute risk of implant failure of $23.4 \%$ in the unreamed group thereby implying a risk difference of $15.9 \%$. The equivalent clinical results in reamed and unreamed groups, even for type IIIB fractures, are possibly explained by no further worsening of the endosteal blood supply in open high-energy fractures where the nutrient artery has been already damaged [6].

Many surgeons have argued for the efficacy of nail dynamization. Georgiadis et al. [16], in a canine model study, found a beneficial effect of dynamization in the quality of early bone but no increase in union rate. Egger et al. [17], using the same animal model, reported an enhancement of the healing process in delayed unions of the tibia but no alteration of union rate. In the current study, the removal of locking screws from either end of the nail was associated with union in $82 \%$ of cases. Despite the scarcity of relevant published clinical data, we advocate conversion of static fixation into a dynamic one, even several months after the index surgical procedure, with the caveat that malalignment or substantial bone loss are not present in the fracture [18].

Locking screw breakage has been considered a "selfdynamization" procedure, which may indicate progression of fracture healing [2]. However, broken screws were usually described in association with unreamed nails and implant failure, malunion or nonunion. In the presented study, 11 out of the 15 fractures with screw breakage were united eventually and only four became nonunions. This suggests that screw failure is not a reliable prognostic factor for fracture nonunion and should be interpreted with caution and taken into account with other parameters such as fracture gap or bone loss.

The increase of intra-compartmental pressures in the lower extremity after reamed intramedullary nailing has been attributed to muscle stretching at the time of fracture reduction, canal reaming and nail insertion $[6,19]$. However, the incidence of compartment syndrome seems to be equal among reamed and unreamed treatment groups $[2,7$, 19]. McQueen et al. [20] demonstrated that delay in time to reamed tibial nailing did not decrease the intra-compartmental pressures or the occurrence of the syndrome.

A dropped hallux after tibial nailing is a rare condition which is probably related to localized compartment syndrome or peroneal nerve damage [6]. Robinson et al. [21] found 11 patients (5.3\%) after IM tibial nailing with dysfunction of the peroneal nerve and dropped hallux but no evidence of compartment syndrome. In our study, two patients $(0.8 \%)$ with a dropped hallux required surgical intervention; scar tissue formation at the musculotendinous area of flexor hallucis longus was observed intraoperatively. This finding confirms that a local compartment syndrome can occur after IM nailing. Due to its subclinical course, early diagnosis is very difficult but a high index of suspicion should be raised in case of weakness of extensor hallucis longus or numbness in the first web space. Further studies are needed to determine the potential role of injury severity, tourniquet application and amount of reaming in the pathogenesis of the syndrome.

The mean incidence of anterior knee pain after tibial nailing is close to $50 \%$ but rates up to $86 \%$ have been also described in the literature [22]. The transtendinous approach has been reported to increase the incidence of anterior knee pain [14] but other studies have not shown a difference between transtendinous and paratendinous approaches [22]. It would appear that, apart from the surgical incision or a nail prominence, other factors may be responsible for the symptom. This was illustrated by the 43 and $67 \%$ of patients, respectively, in the current study and that by Toivanen et al. [22] who still complained of anterior knee pain despite implant removal. Thigh muscle weakness from reflex inhibition of quadriceps and injuries to collateral ligaments, cruciate ligaments, menisci or the articular cartilage can predispose to knee pain after IM nailing [23]. Furthermore, Cartwright et al. [24] noticed a strong correlation of pain between the knees of the injured and the uninjured limb. The authors suggested that a preoperative assessment could predict which patients are more likely to develop knee pain.

Reamed IM nailing remains a "gold standard" for bone healing in aseptic tibial nonunions without substantial bone loss [25]. In exchange nailing, the nail should be at least $1 \mathrm{~mm}$ larger in diameter but nails up to $2-4 \mathrm{~mm}$ larger can be used in order to provide stronger constructs [25]. The technique should be avoided in isolation when large segmental defects or foreshortening of more than $1 \mathrm{~cm}$ exists [25]. Brinker and O'Connor [25] reported that the incidence of fracture healing in aseptic tibial nonunions after exchange nailing ranged from 76 to $96 \%$ from the available published studies. However, to the best of our knowledge, no comparative trials between nailing only and nailing plus 
grafting have been performed. In this study, no difference was found between the above groups but the small number of cases and the absence of fracture categorization do not allow the firm conclusions to be derived.

\section{Conclusion}

Reamed interlocked intramedullary nailing can be safely and effectively performed for the vast majority of acute tibial fractures and aseptic nonunions. Neither nail diameter nor number of locking screws is of primary importance in achieving fracture union in the acute fracture. The available data from this study supports dynamization in fractures with slow progression to healing by selective locking screw removal, but does not advocate routine use of bone grafting in aseptic nonunions.

Conflict of interest statement The authors state that no benefits have been received from a commercial party related directly or indirectly to the subject of this article and they have no conflicts of interest to report.

\section{References}

1. Blachut PA, O'Brien PJ, Meek RN, Broekhuyse HM (1997) Interlocking intramedullary nailing with and without reaming for the treatment of closed fractures of the tibial shaft. A prospective, randomized study. J Bone Joint Surg Am 79:640-646

2. Finkemeier CG, Schmidt AH, Kyle RF, Templeman DC, Varecka $\mathrm{TF}$ (2000) A prospective, randomized study of intramedullary nails inserted with and without reaming for the treatment of open and closed fractures of the tibial shaft. J Orthop Trauma 14:187-193

3. Keating JF, O'Brien PJ, Blachut PA, Meek RN, Broekhuyse HM (1997) Locking intramedullary nailing with and without reaming for open fractures of the tibial shaft. A prospective, randomized study. J Bone Joint Surg Am 79:334-341

4. Muller ME, Allgower M, Schneider R, Willenegger H, SM P (1991) Manual of internal fixation: techniques recommended by the AO-ASIF group. Springer-Verlag, Berlin

5. Gustilo RB, Mendoza RM, Williams DN (1984) Problems in the management of type III (severe) open fractures: a new classification of type III open fractures. J Trauma 24:742-746

6. Court-Brown CM (2004) Reamed intramedullary tibial nailing: an overview and analysis of 1106 cases. J Orthop Trauma 18:96-101

7. Larsen LB, Madsen JE, Hoiness PR, Ovre S (2004) Should insertion of intramedullary nails for tibial fractures be with or without reaming? A prospective, randomized study with 3.8 years' follow-up. J Orthop Trauma 18:144-149

8. Klein MP, Rahn BA, Frigg R, Kessler S, Perren SM (1990) Reaming versus non-reaming in medullary nailing: interference with cortical circulation of the canine tibia. Arch Orthop Trauma Surg 109:314-316

9. Schemitsch EH, Kowalski MJ, Swiontkowski MF, Senft D (1994) Cortical bone blood flow in reamed and unreamed locked intramedullary nailing: a fractured tibia model in sheep. J Orthop Trauma 8:373-382

10. Reichert IL, McCarthy ID, Hughes SP (1995) The acute vascular response to intramedullary reaming. Microsphere estimation of blood flow in the intact ovine tibia. J Bone Joint Surg Br 77:490493

11. Frolke JP, Nulend JK, Semeins CM, Bakker FC, Patka P, Haarman HJ (2004) Viable osteoblastic potential of cortical reamings from intramedullary nailing. J Orthop Res 22:1271-1275

12. Wenisch S, Trinkaus K, Hild A, Hose D, Herde K, Heiss C, Kilian O, Alt V, Schnettler R (2005) Human reaming debris: a source of multipotent stem cells. Bone 36:74-83

13. Court-Brown CM, Keating JF, Christie J, McQueen MM (1995) Exchange intramedullary nailing. Its use in aseptic tibial nonunion. J Bone Joint Surg Br 77:407-411

14. Keating JF, O'Brien PI, Blachut PA, Meek RN, Broekhuyse HM (1997) Reamed interlocking intramedullary nailing of open fractures of the tibia. Clin Orthop Relat Res 338:182-191

15. Bhandari M, Guyatt GH, Swiontkowski MF, Schemitsch EH (2001) Treatment of open fractures of the shaft of the tibia. J Bone Joint Surg Br 83:62-68

16. Georgiadis GM, Minster GJ, Moed BR (1990) Effects of dynamization after interlocking tibial nailing: an experimental study in dogs. J Orthop Trauma 4:323-330

17. Egger EL, Gottsauner-Wolf F, Palmer J, Aro HT, Chao EY (1993) Effects of axial dynamization on bone healing. J Trauma 34:185-192

18. Phieffer LS, Goulet JA (2006) Delayed unions of the tibia. Instr Course Lect 55:389-401

19. Nassif JM, Gorczyca JT, Cole JK, Pugh KJ, Pienkowski D (2000) Effect of acute reamed versus unreamed intramedullary nailing on compartment pressure when treating closed tibial shaft fractures: a randomized prospective study. J Orthop Trauma 14:554-558

20. McQueen MM, Christie J, Court-Brown CM (1990) Compartment pressures after intramedullary nailing of the tibia. $\mathrm{J}$ Bone Joint Surg Br 72:395-397

21. Robinson CM, O’Donnell J, Will E, Keating JF (1999) Dropped hallux after the intramedullary nailing of tibial fractures. J Bone Joint Surg Br 81:481-484

22. Toivanen JA, Vaisto O, Kannus P, Latvala K, Honkonen SE, Jarvinen MJ (2002) Anterior knee pain after intramedullary nailing of fractures of the tibial shaft. A prospective, randomized study comparing two different nail-insertion techniques. J Bone Joint Surg Am 84:580-585

23. Bhattacharyya T, Seng K, Nassif NA, Freedman I (2006) Knee pain after tibial nailing: the role of nail prominence. Clin Orthop Relat Res 449:303-307

24. Cartwright-Terry M, Snow M, Nalwad H (2007) The severity and prediction of anterior knee pain post tibial nail insertion. J Orthop Trauma 21:381-385

25. Brinker MR, O’Connor DP (2007) Exchange nailing of ununited fractures. J Bone Joint Surg Am 89:177-188 